\title{
TOEPLITZ-PLUS-HANKEL BEZOUTIANS AND INVERSES OF TOEPLITZ AND TOEPLITZ-PLUS-HANKEL MATRICES
}

\author{
KARLA ROST
}

\begin{abstract}
In the present paper Bezoutian-type formulas for the inverses of Toeplitz-plus-Hankel $(\mathrm{T}+\mathrm{H})$ matrices are presented which involve bases of kernels of associated rectangular $\mathrm{T}+\mathrm{H}$ matrices. Special Bezoutians of this type yield inverses of symmetric or skewsymmetric Toeplitz matrices and vice versa. In the skewsymmetric case these formulas lead directly to splitting formulas for inverses of centro-skewsymmetric $\mathrm{T}+\mathrm{H}$ matrices.
\end{abstract}

Mathematics subject classification (2000): 65F05, 15A06, 15A09.

Key words and phrases: Toeplitz-plus-Hankel matrix, inversion, Bezoutian.

\section{REFERENCES}

[1] P. Delsarte, Y. Genin, The split Levinson algorithm, IEEE Transactions on Acoustics Speech, and Signal Processing ASSP-34 (1986), pp. 470-477.

[2] P. Delsarte, Y. GENIN, On the splitting of classical algorithms in linear prediction theory, IEEE Transactions on Acoustics Speech, and Signal Processing ASSP-35 (1987), pp. 645-653.

[3] I. GoHBERG, I. KoltraChT, Efficient algorithm for Toeplitz plus Hankel matrices, Integral Equations Operator Theory, 12, 1 (1989), pp. 136-142.

[4] I. Gohberg, A. A. Semencul, On Inversion of finite-section Toeplitz matrices and their continuos analogues (in Russian), Matem. Issled., Kishinev 7, 2 (1972), pp. 201-224.

[5] G. HEINIG, Chebyshev-Hankel matrices and the splitting approach for centrosymmetric Toeplitz-plusHankel matrices, Linear Algebra Appl., 327, 1-3 (2001), pp. 181-196.

[6] G. HEINIG, Kernel structure of Toeplitz-plus-Hankel matrices, Linear Algebra Appl., 340, (2003), pp. $1-13$.

[7] G. HeINIG, K. Rost, Algebraic Methods for Toeplitz-like Matrices and Operators, Birkhäuser Verlag, Basel, Boston, Stuttgart and Akademie-Verlag, Berlin, 1984.

[8] G. HeINIG, K. Rost, On the inverses of Toeplitz-plus-Hankel matrices, Linear Algebra Appl., 106 (1988), pp. 39-52.

[9] G. HEINIG, K. Rost, DFT representations of Toeplitz-plus-Hankel Bezoutians with application to fast matrix-vector multiplication, Linear Algebra Appl., 284 (1998), pp. 157-175.

[10] G. HeINIG, K. Rost, Hartley transform representations of inverses of real Toeplitz-plus-Hankel matrices, Numerical Functional Analysis and Optimization, 21 (2000), pp. 175-189.

[11] G. HeINIG, K. Rost, Representations of inverses of real Toeplitz-plus-Hankel matrices using trigonometric transformations, In: Large-scale scientific computations of engineering and environmental problems, II (Sozopol, 1999), Vieweg, Braunschweig (2000), pp. 80-86.

[12] G. HEINIG, K. Rost, Efficient inversion formulas for Toeplitz-plus-Hankel matrices using trigonometric transformations, In: V. OLSHEVSKY (Ed.), Structured Matrices in Mathematics, Computer Science, and Engeneering, vol. 2, AMS-Series Contemporary Mathematics, vol. 281 (2001), pp. 247-264.

[13] G. HeINIG, K. Rost, Fastalgorithms for skewsymmetric Toeplitz matrices, In: A. BÖTTCHER, I. GoHBERG, P. JUNGHANNS, Toeplitz matrices and singular integral equations, Operator Theory: Advances and Applications, vol. 135, Birkhäuser Verlag, Basel, Boston, Berlin (2002), pp. 193-208.

[14] G. HeInIG, K. Rost, Centrosymmetric and centro-skewsymmetric Toeplitz-plus-Hankel matrices and Bezoutians, Linear Algebra Appl., 366 (2003), pp. 257-281. 
[15] G. HeINIG, K. Rost, Fast algorithms for centro-symmetric and centro-skewsymmetric Toeplitz-plusHankel matrices, Numerical Algorithms, 33 (2003), pp. 305-317.

[16] G. HeINIG, K. Rost, Split algorithms for skewsymmetric Toeplitz matrices with arbitrary rank profile, Theoretical Computer Science, 315, 2-3, (2004), pp. 453-468.

[17] A. MELMAN, The even-odd split Levinson Algorithm for Toeplitz systems, SIAM J. Matrix Anal. Appl., 23, 1 (2001), pp. 256-270.

[18] G. A. MERChant, T. W. PARKs, Efficient solution of a Toeplitz-plus-Hankel coefficient matrix system of equations, IEEE Transact. on ASSP, 30, 1 (1982), pp. 40-44. 